\title{
Simple Proofs of Classical Theorems in Discrete Geometry via the Guth-Katz Polynomial Partitioning Technique*
}

\author{
HAIM KAPLAN \\ School of Computer Science, \\ Tel Aviv University, \\ Tel Aviv 69978, Israel \\ JiŘí MATOUŠEK \\ Department of Applied Mathematics and \\ Institute of Theoretical Computer Science (ITI) \\ Charles University, Malostranské nám. 25 \\ 11800 Praha 1, Czech Republic, and \\ Institute of Theoretical Computer Science \\ ETH Zurich, 8092 Zurich, Switzerland \\ MiCHA SHARIR \\ School of Computer Science, \\ Tel Aviv University, \\ Tel Aviv 69978, Israel, and \\ Courant Institute of Mathematical Sciences, \\ New York University, \\ New York, NY 10012, USA
}

March 1, 2011

\begin{abstract}
Recently Guth and Katz 16 invented, as a step in their nearly complete solution of Erdös's distinct distances problem, a new method for partitioning finite point sets in $\mathbb{R}^{d}$, based on the Stone-Tukey polynomial ham-sandwich theorem. We apply this method to obtain new and simple proofs of two well known results: the Szemerédi-Trotter theorem on incidences of points and lines, and the existence of spanning trees with low crossing numbers. Since we consider these proofs particularly suitable for teaching, we aim at self-contained, expository treatment. We also mention some generalizations and extensions, such as the Pach-Sharir bound on the number of incidences with algebraic curves of bounded degree.
\end{abstract}

\section{Introduction}

A dramatic breakthrough in discrete geometry took place in November 2010, when Guth and Katz [16] completed a project of Elekes, exposed in [13, and established a nearly complete solution of Erdös's distinct distances problem [14], originally posed in 1946.

In one of the main steps of their analysis, they apply the polynomial ham-sandwich theorem of Stone and Tukey [33. to obtain a partition of a finite point set $P$ in $\mathbb{R}^{d}$ with certain favorable properties, detailed in Section 2.3 below. The partition is effected by what we call an $r$-partitioning

\footnotetext{
*Work by Haim Kaplan has been supported by Grant 2006/204 from the U.S.-Israel Binational Science Foundation, and by grant 822/10 from the Israel Science Fund. Work by Micha Sharir has been supported by NSF Grant CCF08-30272, by Grant 2006/194 from the U.S.-Israel Binational Science Foundation, by grant 338/09 from the Israel Science Fund, and by the Hermann Minkowski-MINERVA Center for Geometry at Tel Aviv University.
} 
polynomial. The removal of the zero set $Z$ of the polynomial partitions space into connected components, each containing at most $|P| / r$ points of $P$. A key feature of the construction is that the degree of the polynomial achieving this need not be too high, only $O\left(r^{1 / d}\right)$, and thus the interaction of other objects, such as lines or hyperplanes, with $Z$ is under control in some sense.

In this paper we apply partitioning polynomials in several classical problems of discrete geometry, mostly planar ones, and we provide new and simple proofs of some well known results.

Incidences. For a finite set $P \subset \mathbb{R}^{2}$ and a finite set $L$ of lines in $\mathbb{R}^{2}$, let $I(P, L)$ denote the number of incidences of $P$ and $L$, i.e., of pairs $(p, \ell)$ with $p \in P, \ell \in L$, and $p \in \ell$.

The following fundamental result was first proved by Szemerédi and Trotter in 1983, in response to a problem of Erdős [14.

Theorem 1.1 (Szemerédi and Trotter [35]) $I(P, L)=O\left(m^{2 / 3} n^{2 / 3}+m+n\right)$ for every set $P$ of $m$ distinct points in the plane and every set $L$ of $n$ distinct lines.

We remark that the bound in the theorem is tight in the worst case for all $m, n$ (see [14], [1] for original sources or [24] for a presentation).

A simpler proof of the Szemerédi-Trotter theorem, based on cuttings, was given by Clarkson et al. 8] in 1990, and in 1997 Székely [34] found a beautiful and elegant proof, based on the crossing lemma for graphs embedded in the plane (also see, e.g., [24]).

In Section 3 we present an alternative proof based on polynomial partitions, hoping that the reader will find it equally simple. We also believe that the new proof is suitable for teaching purposes, so our goal is to make the exposition as elementary and self-contained as possible. For this we also give proofs of several well known and basic facts about multivariate polynomials. The only major ingredient of the analysis which we do not prove is the classical ham-sandwich theorem, which we use as a black box (see, e.g., 25] for an exposition).

The Szemerédi-Trotter theorem has led to an extensive study of incidences of points and curves in the plane and of points and surfaces in higher dimensions. A survey of the topic can be found in Pach and Sharir [30]. In particular, the following theorem on incidences between points and planar curves has been established:

Theorem 1.2 (Pach and Sharir [29]) Let $P$ be a set of $m$ points and let $\Gamma$ be a set of $n$ simple curves, all lying in the plane. If no more than $C_{1}$ curves of $\Gamma$ pass through any $k$ given points, and every pair of curves of $\Gamma$ intersect in at most $C_{2}$ points, then

$$
I(P, \Gamma)=O\left(m^{k /(2 k-1)} n^{(2 k-2) /(2 k-1)}+m+n\right),
$$

with an appropriate constant of proportionality that depends on $k, C_{1}, C 2$.

A weaker version of this result, where the the curves in $\Gamma$ are assumed to be algebraic and to belong to a family parameterized by $k$ real parameters, was obtained earlier, also by Pach and Sharir [28] (special cases of this result, e.g., for incidences of points and circles, were obtained even earlier by Clarkson et al. [8]).

In Section 4, we give a simple proof of a version of Theorem 1.2, with the additional assumption that $\Gamma$ consists of algebraic curves of degree bounded by a constant. 
Spanning trees with low crossing number. Let $P$ be a finite set of points in $\mathbb{R}^{2}$. A (geometric) graph on $P$ is a graph $G$ with vertex set $P$ whose edges are realized as straight segments connecting the respective end-vertices. The crossing number of $G$ is the maximum number of edges that can be intersected simultaneously by a line not passing through any point of $P 1$ We will consider geometric spanning trees on $P$, i.e., acyclic connected geometric graphs on $P$.

The following result has been established in the late 1980s by Welzl [38] and by Chazelle and Welzl [7]; also see Welzl [39].

Theorem 1.3 (Welzl [38], Chazelle and Welzl [7]) Every set of $n$ points in the plane has a geometric spanning tree with crossing number $O(\sqrt{n})$.

The bound in the theorem is tight up to a multiplicative constant, as the example of a $\sqrt{n} \times \sqrt{n}$ grid shows. Spanning trees with low crossing number have many applications in discrete and computational geometry, including range searching [7, the design of other geometric algorithms (see, e.g., 3]), discrepancy theory [26], and approximation [23].

The original proof of Theorem 1.3 constructs the tree iteratively, through a process called iterative reweighting. In each step several new edges are added, and these are selected using a packing argument with balls in a line arrangement (or, alternatively, using a so-called cutting). An alternative proof, replacing iterative reweighting with linear programming duality, was recently given by Har-Peled [18.

In Section 5 we present a new and simple proof of Theorem 1.3 via polynomial partitions.

Chazelle and Welzl [7] established their result on spanning trees with low crossing number in a very general setting, where the points do not lie in the plane, but rather in the ground set of an arbitrary set system $\mathcal{F}$. The bound on the crossing number is then expressed in terms of the dual shatter function of $\mathcal{F}$.

At present it seems that the approach with polynomial partitions is not suitable for this level of generality. However, some generalizations are possible. First, we have verified that Theorem 1.3 can be extended to the case where the crossing number is taken with respect to a family of algebraic curves of degree bounded by a constant, but we will not pursue this in this paper.

Second, one can also prove a $d$-dimensional generalization of Theorem 1.3, and this we do in Section 6. Here we are given a set $P$ of $n$ points in $\mathbb{R}^{d}$, and consider spanning trees of $P$, which we embed into $\mathbb{R}^{d}$ by drawing their edges as straight segments connecting the respective end-vertices, as in the plane. The crossing number of such a tree is the maximum number of its edges that are crossed by a hyperplane not passing through any point of $P$. According to [38, [7, for every $n$-point set in $\mathbb{R}^{d}$ there exists a straight-edge spanning tree with crossing number $O\left(n^{1-1 / d}\right)$.

We re-prove this fact using polynomial partitions, similar to the planar case. However, the proof is more involved in higher dimensions. Informally, the partition distributes the input points evenly among the resulting cells, except that some (in the worst case even all) of the points may lie on the zero set $Z$ of the partitioning polynomial, and therefore not belong to any of the subsets.

We avoid this situation using a perturbation argument. This works for the spanning tree construction because there we may assume general position of the input points. For incidence problems this assumption cannot be made, and other techniques are needed to handle the points on $Z$. We intend to investigate alternative approaches to handling points on $Z$ in a subsequent paper.

\footnotetext{
${ }^{1}$ The condition of avoiding the points of $P$ is important; for example, if all of the points of $P$ are collinear, then the line containing $P$ necessarily intersects all edges.
} 


\section{Review of tools}

\subsection{Preliminaries on polynomials}

Here we recall some standard facts about polynomials. The proofs are given for didactic purposes, and can be skipped by more experienced readers.

Since most of the problems that we study here are planar, we will consider mostly bivariate polynomials $f=f(x, y)=\sum_{i, j} a_{i j} x^{i} y^{j} \in \mathbb{R}[x, y]$, but the analysis can easily be extended to $d$ variate polynomials in $\mathbb{R}^{d}$. The degree of $f$ is $\operatorname{deg}(f)=\max \left\{i+j \mid a_{i j} \neq 0\right\}$. Let $Z(f)=\{(x, y) \in$ $\left.\mathbb{R}^{2} \mid f(x, y)=0\right\}$ denote the zero set of $f$.

Lemma 2.1 If $\ell$ is a line in $\mathbb{R}^{2}$ and $f \in \mathbb{R}[x, y]$ is of degree at most $D$, then either $\ell \subseteq Z(f)$, or $|\ell \cap Z(f)| \leq D$.

Proof. Writing $\ell$ in parametric form $\left\{\left(u_{1} t+v_{1}, u_{2} t+v_{2}\right) \mid t \in \mathbb{R}\right\}$, we get that the points of $\ell \cap Z(f)$ are roots of the univariate polynomial $g(t):=f\left(u_{1} t+v_{1}, u_{2} t+v_{2}\right)$, which is of degree at most $D$. Thus, either $g$ is identically 0 , or it has at most $D$ roots.

Lemma 2.2 If $f \in \mathbb{R}[x, y]$ is nonzero and of degree at most $D$, then $Z(f)$ contains at most $D$ distinct lines.

Proof. We need to know that a nonzero bivariate polynomial (i.e., with at least one nonzero coefficient) does not vanish on all of $\mathbb{R}^{2}$. (Readers who do not consider this a sufficiently standard fact are welcome to work out a quick proof.)

Now we fix a point $p \in \mathbb{R}^{2}$ not belonging to $Z(f)$. Let us suppose that $Z(f)$ contains lines $\ell_{1}, \ldots, \ell_{k}$. We choose another line $\ell$ passing through $p$ that is not parallel to any $\ell_{i}$ and not passing through any of the intersections $\ell_{i} \cap \ell_{j}$. (Such an $\ell$ exists since only finitely many directions need to be avoided.) Then $\ell$ is not contained in $Z(f)$ and it has $k$ intersections with $\bigcup_{i=1}^{k} \ell_{i}$. Lemma 2.1 yields $k \leq D$.

In the proof of Theorem 1.3 (spanning trees with low crossing number), we will also need the following result.

Theorem 2.3 (Harnack's curve theorem [17]) Let $f \in \mathbb{R}[x, y]$ be a bivariate polynomial of degree $D$. Then the number of (arcwise) connected components of $Z(f)$ is at most $1+\left(\begin{array}{c}D-1 \\ 2\end{array}\right)$. The bound is tight in the worst case.

For our application, we actually do not need the precise bound in Harnack's theorem; it suffices to know that the number of components is at most $O\left(D^{2}\right)$. For the sake of completeness, we provide a short proof of an almost tight bound.

First we recall, without proof, another basic result in algebraic geometry; see, e.g., [4, 9, 10].

Theorem 2.4 (Bézout's theorem) Let $f, g \in \mathbb{R}[x, y]$ be two bivariate polynomials of degrees $D_{f}$ and $D_{g}$, respectively. (a) If the system $f=g=0$ has finitely many solutions, then their number is at most $D_{f} D_{g}$. (b) If the system $f=g=0$ has infinitely many solutions, then $f$ and $g$ have $a$ nontrivial common factor. 
For a proof of Theorem 2.3, we choose a generic direction, and assume, without loss of generality, that it is the $x$-direction. We may assume that $f$ is square-free, because eliminating repeated factors of $f$ does not change its zero set.

Every bounded component of $Z(f)$ has at least two extreme points in the $x$-direction (that is, its leftmost and rightmost points). Such an extreme point has to satisfy $f=f_{y}=0$, where $f_{y}$ is the partial derivative of $f$ with respect to $y$.

Since $f$ is square-free, $f$ and $f_{y}$ have no common factor 2 and so by Theorem 2.4 the system $f=f_{y}=0$ has at most $D(D-1)$ solutions. Every bounded component consumes at least two of these critical points, and hence the number of bounded components is at most $\frac{1}{2} D(D-1)$.

If $B$ is a sufficiently large number, then (again, assuming generic directions of the coordinate axes) every unbounded component of $Z(f)$ meets (at least) one of the two lines $x=+B$ and $x=-B$. Thus, there are at most $2 D$ unbounded components, and in total we get a bound of $\frac{1}{2} D(D+1)$ on all components.

\subsection{The polynomial ham-sandwich theorem}

Here we review the polynomial ham-sandwich theorem of Stone and Tukey [33], the key tool used by Guth and Katz in constructing their partitioning polynomials.

We assume the standard ham-sandwich theorem in the following discrete version: Every $d$ finite sets $A_{1}, \ldots, A_{d} \subset \mathbb{R}^{d}$ can be simultaneously bisected by a hyperplane. Here a hyperplane $h$ bisects a finite set $A$ if neither of the two open halfspaces bounded by $h$ contains more than $\lfloor|A| / 2\rfloor$ points of $A$.

From this, it is easy to derive the polynomial ham-sandwich theorem, which we state for bivariate polynomials.

Theorem 2.5 Let $A_{1}, \ldots, A_{s} \subseteq \mathbb{R}^{2}$ be finite sets, and let $D$ be an integer such that $\left(\begin{array}{c}D+2 \\ 2\end{array}\right)-1 \geq s$. Then there exists a nonzero polynomial $f \in \mathbb{R}[x, y]$ of degree at most $D$ that simultaneously bisects all the sets $A_{i}$, where " $f$ bisects $A_{i}$ " means that $f>0$ in at most $\left\lfloor\left|A_{i}\right| / 2\right\rfloor$ points of $A_{i}$ and $f<0$ in at most $\left\lfloor\left|A_{i}\right| / 2\right\rfloor$ points of $A_{i}$.

Proof. We note that $\left(\begin{array}{c}D+2 \\ 2\end{array}\right)$ is the number of monomials in a bivariate polynomial of degree $D$, or in other words, the number of pairs $(i, j)$ of nonnegative integers with $i+j \leq D$. We set $k:=\left(\begin{array}{c}D+2 \\ 2\end{array}\right)-1$, and we let $\Phi: \mathbb{R}^{2} \rightarrow \mathbb{R}^{k}$ denote the Veronese map, given by

$$
\Phi(x, y):=\left(x^{i} y^{j}\right)_{(i, j) \mid 1 \leq i+j \leq D} \in \mathbb{R}^{k} .
$$

(We think of the coordinates in $\mathbb{R}^{k}$ as indexed by pairs $(i, j)$ with $1 \leq i+j \leq D$.)

Assuming, as we may, that $s=k$, we set $A_{i}^{\prime}:=\Phi\left(A_{i}\right), i=1,2, \ldots, k$, and we let $h$ be a hyperplane simultaneously bisecting $A_{1}^{\prime}, \ldots, A_{k}^{\prime}$. Then $h$ has an equation of the form $a_{00}+$ $\sum_{i, j} a_{i j} z_{i j}=0$, where $\left(z_{i j}\right)_{(i, j) \mid 1 \leq i+j \leq d}$ are the coordinates in $\mathbb{R}^{k}$. It is easy to check that $f(x, y):=$ $\sum_{i, j} a_{i j} x^{i} y^{j}$ is the desired polynomial (where here the sum includes $a_{00}$ too).

\footnotetext{
${ }^{2}$ Assume by induction that this is true for polynomials of degree smaller than $D$, and let $f$ be a square-free polynomial of degree $D$. Assume that $f=h \cdot g$ and $f_{y}=h \cdot k$ for some polynomials $h, g$, and $k$, where $h$ is not a constant. Then $f_{y}=h_{y} \cdot g+g_{y} \cdot h=h \cdot k$. So $h$ divides $h_{y} \cdot g$. By induction, $h$ and $h_{y}$ have no common factors, and so $h$ divides $g$, contradicting our assumption that $f$ is square-free.
} 


\subsection{Partitioning polynomials}

In this section we recall the construction of Guth and Katz [16], specialized to the planar setting (our formulation is slightly different from theirs). We also (informally) compare it to older tools of discrete geometry, such as cuttings.

Let $P$ be a set of $n$ points in the plane, and let $r$ be a parameter, $1<r \leq n$. We say that $f \in \mathbb{R}[x, y]$ is an $r$-partitioning polynomial for $P$ if no connected component of $\mathbb{R}^{2} \backslash Z(f)$ contains more than $n / r$ points of $P$.

In the sequel, we will sometimes call the connected components of $\mathbb{R}^{2} \backslash Z(f)$ cells. Let us also stress that the cells are open sets. The points of $P$ lying on $Z(f)$ do not belong to any cell, and usually they require a special treatment.

Theorem 2.6 (Polynomial partitioning theorem) For every $r>1$, every finite point set $P \subset$ $\mathbb{R}^{2}$ admits an $r$-partitioning polynomial $f$ of degree at most $O(\sqrt{r})$.

Proof. We inductively construct collections $\mathcal{P}_{0}, \mathcal{P}_{1}, \ldots$, each consisting of disjoint subsets of $P$, such that $\left|\mathcal{P}_{j}\right| \leq 2^{j}$ for each $j$. We start with $\mathcal{P}_{0}:=\{P\}$. Having constructed $\mathcal{P}_{j}$, with at most $2^{j}$ sets, we use the polynomial ham-sandwich theorem to construct a polynomial $f_{j}$, of degree $\operatorname{deg}\left(f_{j}\right) \leq \sqrt{2 \cdot 2^{j}}$, that bisects each of the sets of $\mathcal{P}_{j}$. Then for every subset $Q \in \mathcal{P}_{j}$, we let $Q^{+}$ consist of the points of $Q$ at which $f_{j}>0$, and let $Q^{-}$consist of the points of $Q$ with $f_{j}<0$, and we put $\mathcal{P}_{j+1}:=\bigcup_{Q \in \mathcal{P}_{j}}\left\{Q^{+}, Q^{-}\right\}$.

Each of the sets in $\mathcal{P}_{j}$ has size at most $|P| / 2^{j}$. We let $t=\left\lceil\log _{2} r\right\rceil$; then each of the sets in $\mathcal{P}_{t}$ has size at most $|P| / r$. We set $f:=f_{1} f_{2} \cdots f_{t}$.

By the construction, no component of $\mathbb{R}^{2} \backslash Z(f)$ can contain points of two different sets in $\mathcal{P}_{t}$, because any arc connecting a point in one subset to a point in another subset must contain a point at which one of the polynomials $f_{j}$ vanishes, so the arc must cross $Z(f)$. Thus $f$ is an $r$-partitioning polynomial for $P$.

It remains to bound the degree:

$$
\operatorname{deg}(f)=\operatorname{deg}\left(f_{1}\right)+\operatorname{deg}\left(f_{2}\right)+\cdots+\operatorname{deg}\left(f_{t}\right) \leq \sqrt{2} \sum_{j=1}^{t} 2^{j / 2} \leq \frac{2}{\sqrt{2}-1} 2^{t / 2} \leq c \sqrt{r} .
$$

where $c=2 \sqrt{2} /(\sqrt{2}-1)<7$.

A comparison with other partitioning techniques 3 The Guth-Katz technique with partitioning polynomials is useful for problems where we deal with a finite point set $P$ and with a collection $\Gamma$ of lines, algebraic curves, or algebraic varieties in higher dimensions. It provides a method of implementing the divide-and-conquer paradigm.

In the planar case discussed above, the plane is subdivided by $Z(f)$ into some number of (open, connected) cells, each containing at most $|P| / r$ points of $P$. If $\Gamma$ consists of lines, then every $\gamma \in \Gamma$ intersects at most $\operatorname{deg}(f)+1=O(\sqrt{r})$ cells (by Lemma 2.1). Similarly, for $\Gamma$ consisting of algebraic curves of degree bounded by a constant, every $\gamma \in \Gamma$ intersects at most $O(\sqrt{r})$ cells by Bézout's theorem (Theorem 2.4). Thus, if we define, for every cell $C_{i}$ of $\mathbb{R}^{2} \backslash Z(f)$, a subset $P_{i} \subseteq P$ as the

\footnotetext{
${ }^{3}$ This part is slightly more advanced and assumes some familiarity with previous techniques used in incidence problems.
} 
set of points of $P$ contained in $C_{i}$, and we let $\Gamma_{i}$ consist of the lines or curves of $\Gamma$ intersecting $C_{i}$, then $\left|P_{i}\right| \leq|P| / r$ for all $i$, and the average size of the $\Gamma_{i}$ is $O(|\Gamma| / \sqrt{r})$.

There are two earlier partitioning tools in discrete geometry with a similar effect. The first, and simpler, kind of them are cuttings (see [6]). A cutting for a collection $\Gamma$ of curves in the plane subdivides $\mathbb{R}^{2}$ into a collection of connected, simply shaped cells, in such a way that no cell is crossed by more than a prescribed fraction of the curves of $\Gamma$. If we again let $P_{i}$ denote the set of points of $P$ in the $i$ th cell, and $\Gamma_{i}$ is the set of the curves intersecting that cell, then this time all $\Gamma_{i}$ have size $O(|\Gamma| / \sqrt{r})$, and the average of the sizes $\left|P_{i}\right|$ i. $|P| / r$. Thus, the behavior of cuttings is, in a sense, "dual" to that of polynomial partitions. For many applications, this does not really make a difference.

The second of the earlier tools are simplicial partitions [22. Here, as in the case of polynomial partitions, the plane is subdivided into cells so that $\left|P_{i}\right| \leq|P| / r$ for each $i$ (where, again, $P_{i}$ is the set of points of $P$ in the $i$ th cell), and no $\gamma \in \Gamma$ intersects more than $O(\sqrt{r})$ cells 5

In the plane, as far as we can see, whatever can be done with polynomial partitions, can also be achieved through cuttings or through simplicial partitions. The main advantage of polynomial partitions is simplicity of the proof. On the other hand, cuttings and simplicial partitions can be constructed and manipulated with fairly efficient algorithms, at least in the sense of asymptotic complexity, which is not at all clear for polynomial partitions. (For example, finding a hamsandwich cut in a high-dimensional space is a rather costly operation; see 20] for a computational hardness result and references.)

Polynomial partitions may be more powerful than the earlier tools if we pass to a higherdimensional space $\mathbb{R}^{d}, d>2$. Asymptotically optimal cuttings and simplicial partitions are known to exist in $\mathbb{R}^{d}$, for every fixed $d$, in the case where $\Gamma$ is a collection of hyperplanes. However, if we want to apply analogous methods to construct cuttings (or simplicial partitions, whose construction needs cuttings as a subroutine) for $\Gamma$ consisting of algebraic surfaces of degree bounded by a constant, say, then there is a stumbling block. In one of the steps of the construction, we have a collection $\Gamma^{\prime}$ of $m$ surfaces from $\Gamma$. It is known that these surfaces partition $\mathbb{R}^{d}$ into $O\left(m^{d}\right)$ cells, but we need to further subdivide each cell into subcells, so that each of the resulting subcells can be described by a constant number of real parameters. There is no known general solution that achieves $O\left(\mathrm{~m}^{d}\right)$ subcells in total, which is the optimal bound one is after for most applications. For $d=3,4$, the situation is still not bad, since bounds only slightly worse than $O\left(m^{d}\right)$ have been proved, but for $d \geq 5$, the best bound is of order roughly $m^{2 d-4}$, and so for large $d$, the exponent is almost twice larger of what it probably should be (see [1] for a more detailed discussion). The new approach with polynomial partitions might hopefully be able to bypass this stumbling block, at least in non-algorithmic applications.

\section{Proof of the Szemerédi-Trotter theorem}

We recall that we are given a set $P$ of $m$ distinct points and a set $L$ of $n$ distinct lines in the plane and we want to bound the number of incidences $I(P, L)$.

We begin with a simple observation (appearing in most of the previous proofs).

\footnotetext{
${ }^{4}$ Here we choose the parameterization so that it agrees with the one for polynomial partitions; the usual notation in the literature would use $r$ for our $\sqrt{r}$.

${ }^{5}$ In the original version of simplicial partitions 22 , the cells cover $\mathbb{R}^{2}$, but they need not be disjoint. In a newer version due to Chan [5], disjointness can also be guaranteed.
} 
Lemma $3.1 I(P, L) \leq n+m^{2}$.

Proof. We divide the lines of $L$ into two subsets: the lines in $L^{\prime}$ are incident to at most one point of $P$, while the lines in $L^{\prime \prime}$ pass through at least two points.

Obviously, $I\left(P, L^{\prime}\right) \leq\left|L^{\prime}\right| \leq n$. In order to bound $I\left(P, L^{\prime \prime}\right)$, we note that a point $p \in P$ may have at most $m-1$ incidences with the lines of $L^{\prime \prime}$, since there are at most $m-1$ lines passing through $p$ and some other point of $P$. Thus, $I\left(P, L^{\prime \prime}\right) \leq m(m-1) \leq m^{2}$.

Let us remark that this lemma also follows from the Kövári-Sós-Turán theorem [21] concerning graphs with forbidden complete bipartite subgraphs. In the above argument, we are really proving the required instance of Kővári-Sós-Turán.

Proof of the Szemerédi-Trotter theorem. For simplicity, we first do the proof for $m=n$, and then indicate the changes needed to handle an arbitrary $m$.

We set $r:=n^{2 / 3}$, and we let $f$ be an $r$-partitioning polynomial for $P$. By the polynomial partitioning theorem (Theorem 2.6), we may assume $D=\operatorname{deg}(f)=O(\sqrt{r})=O\left(n^{1 / 3}\right)$.

Let $Z:=Z(f)$, let $C_{1}, \ldots, C_{s}$ be the connected components of $\mathbb{R}^{2} \backslash Z$, let $P_{i}:=P \cap C_{i}$, and let $P_{0}:=P \cap Z$. Since $f$ is an $r$-partitioning polynomial, we have $\left|P_{i}\right| \leq n / r=n^{1 / 3}, i=1,2, \ldots, s$. Furthermore, let $L_{0} \subset L$ consist of the lines of $L$ contained in $Z$; we have $\left|L_{0}\right| \leq D$ by Lemma 2.2.

We decompose

$$
I(P, L)=I\left(P_{0}, L_{0}\right)+I\left(P_{0}, L \backslash L_{0}\right)+\sum_{i=1}^{s} I\left(P_{i}, L\right) .
$$

We can immediately bound

$$
I\left(P_{0}, L_{0}\right) \leq\left|L_{0}\right| \cdot\left|P_{0}\right| \leq\left|L_{0}\right| n \leq D n=O\left(n^{4 / 3}\right),
$$

and

$$
I\left(P_{0}, L \backslash L_{0}\right) \leq\left|L \backslash L_{0}\right| D=O\left(n^{4 / 3}\right),
$$

since each line of $L \backslash L_{0}$ intersects $Z$, and thus also $P_{0}$, in at most $D=\operatorname{deg}(f)$ points.

It remains to bound $\sum_{i=1}^{s} I\left(P_{i}, L\right)$. Let $L_{i} \subset L$ be the set of lines containing at least one point of $P_{i}$ (the $L_{i}$ are typically not disjoint). By Lemma 3.1 we get

$$
\sum_{i=1}^{s} I\left(P_{i}, L_{i}\right) \leq \sum_{i=1}^{s}\left(\left|L_{i}\right|+\left|P_{i}\right|^{2}\right)
$$

We have $\sum_{i=1}^{s}\left|L_{i}\right|=O((D+1) n)=O\left(n^{4 / 3}\right)$, since by Lemma 2.1, no line intersects more than $D+1$ of the sets $P_{i}$. Finally, $\sum_{i=1}^{s}\left|P_{i}\right|^{2} \leq\left(\max _{i}\left|P_{i}\right|\right) \cdot \sum_{i=1}^{s}\left|P_{i}\right| \leq \frac{n}{r} \cdot n=O\left(n^{4 / 3}\right)$. This finishes the proof for the case $m=n$.

We generalize the proof for an arbitrary $m$ as follows. We may assume, without loss of generality, that $m \leq n$; the complementary case is handled by interchanging the roles of $P$ and $L$, via a standard planar duality. We may also assume that $\sqrt{n} \leq m$, since otherwise, the theorem follows from Lemma 3.1. Then we set $r:=m^{4 / 3} / n^{2 / 3}$. Noting that $1 \leq r \leq m$ for the assumed range of $m$, we then proceed as in the case $m=n$ above. We get $D=\operatorname{deg}(f)=O\left(m^{2 / 3} / n^{1 / 3}\right)$, and we check that all the partial bounds in the proof are at most $O\left(m^{2 / 3} n^{2 / 3}\right)$. 


\section{Incidences of points with algebraic curves}

As was announced in the introduction, we prove the following (weaker) version of Theorem 1.2 .

Theorem 4.1 Let $b, k$ and $C$ be constants, let $P$ be a set of $m$ points in the plane, and let $\Gamma$ be $a$ family of planar curves such that

(i) every $\gamma \in \Gamma$ is an algebraic curve of degree at most b, and

(ii) for every $k$ distinct points in the plane, there exist at most $C$ distinct curves in $\Gamma$ passing through all of them.

Then $I(P, \Gamma)=O\left(m^{k /(2 k-1)} n^{(2 k-2) /(2 k-1)}+m+n\right)$, with the constant of proportionality depending on $b, k, C$.

In the proof, we may assume that the curves in $\Gamma$ are irreducible 6 Indeed, if it is not the case, we apply the forthcoming analysis to the irreducible components of the curves of $\Gamma$, whose number is at most $b m$.

We begin with an analog of Lemma 3.1

Lemma 4.2 Under the conditions of Theorem 4.1. we have $I(P, \Gamma)=O\left(n+m^{k}\right)$, and also $I(P, \Gamma)=O\left(m+n^{2}\right)$; the constants of proportionality depend on $b, k, C$.

Proof. For the first estimate, we distinguish between the curves with fewer than $k$ incidences, which altogether generate $O(n)$ incidences, and curves with at least $k$ incidences, observing that there are at most $C\left(\begin{array}{c}m-1 \\ k-1\end{array}\right)$ such curves through each point of $P$.

For the second estimate, we first note that, by the assumed irreducibility and by Bézout's theorem (Theorem 2.4), every pair of curves of $\Gamma$ intersect in at most $b^{2}$ points. Then we distinguish between points lying on at most one curve each, which have $O(m)$ incidences altogether, and the remaining points, each lying on at least two curves. Now a single $\gamma \in \Gamma$ has at most $b^{2}(n-1)$ intersections with the other curves, and thus it contributes at most $b^{2}(n-1)$ incidences with these latter points. So $I(P, \Gamma)=O\left(m+n^{2}\right)$ follows.

Proof of Theorem 4.1. We may assume $m \leq n^{2}$ and $n \leq m^{k}$, for otherwise, the bounds of Lemma 4.2 give $I(P, \Gamma)=O(m+n)$.

We set $r:=m^{2 k /(2 k-1)} / n^{2 /(2 k-1)}$, and we observe that our assumptions on $m, n$ yield $1 \leq r \leq m$. Let $f$ be an $r$-partitioning polynomial for $P$, of degree

$$
\operatorname{deg}(f)=O(\sqrt{r})=O\left(m^{k /(2 k-1)} / n^{1 /(2 k-1)}\right) .
$$

The proof now continues in much the same way as the proof of the Szemerédi-Trotter theorem.

We put $Z:=Z(f)$, let $P_{0}:=P \cap Z$, and let $\Gamma_{0} \subset \Gamma$ consist of the curves fully contained in $Z$. Since every $\gamma \in \Gamma_{0}$ is irreducible, it must be a zero set of a factor of $f$ (this follows from Bézout's theorem), and so $\left|\Gamma_{0}\right| \leq \operatorname{deg}(f)=O(\sqrt{r})$. Hence $I\left(P_{0}, \Gamma_{0}\right)=O\left(m+\left|\Gamma_{0}\right|^{2}\right)=O(m+r)=O(m)$ by the second bound of Lemma 4.2. (Here the argument differs from the one for the Szemerédi-Trotter

\footnotetext{
${ }^{6}$ We recall that a planar algebraic curve $\gamma$ is irreducible if $\gamma=Z(g)$ for an irreducible polynomial $g$, i.e., one that cannot be written as $g=g_{1} g_{2}$ with both $g_{1}, g_{2}$ nonconstant (and real in our case). For $\gamma=Z(g)$ with $g$ arbitrary, we can write $g=g_{1} g_{2} \cdots g_{k}$ as a product of irreducible factors, and the irreducible components of $g$ are $Z\left(g_{1}\right), \ldots, Z\left(g_{k}\right)$.
} 
theorem - in the latter, it was sufficient to use the trivial bound $I\left(P_{0}, L_{0}\right) \leq\left|P_{0}\right| \cdot\left|L_{0}\right|$, which in general is not sufficient here.)

Next, we consider $\gamma \in \Gamma \backslash \Gamma_{0}$. Applying Bézout's theorem to $\gamma$ and every irreducible component of $Z$ in turn, we see that $|\gamma \cap Z| \leq b \cdot \operatorname{deg}(f)=O(\sqrt{r})$. So $I\left(P_{0}, \Gamma \backslash \Gamma_{0}\right)=O(n \sqrt{r})=$ $O\left(m^{k /(2 k-1)} n^{(2 k-2) /(2 k-1)}\right)$.

Letting $C_{1}, \ldots, C_{s}$ be the connected components of $\mathbb{R}^{2} \backslash Z$, it remains to bound $\sum_{i=1}^{s} I\left(P_{i}, \Gamma_{i}\right)$, where $P_{i}=P \cap C_{i}$ and $\Gamma_{i}$ is the set of curves meeting $C_{i}$. By Bézout's theorem once again, we have $\sum_{i=1}^{s}\left|\Gamma_{i}\right|=O(n \cdot \operatorname{deg}(f))=O(n \sqrt{r})$. Then, by the first bound of Lemma 4.2, we obtain

$$
\begin{aligned}
\sum_{i=1}^{s} I\left(P_{i}, \Gamma_{i}\right) & =O\left(\sum_{i=1}^{s}\left(\left|\Gamma_{i}\right|+\left|P_{i}\right|^{k}\right)\right) \leq O(n \sqrt{r})+\left(\max _{i}\left|P_{i}\right|\right)^{k-1} O\left(\sum_{i=1}^{s}\left|P_{i}\right|\right) \\
& =O\left(n \sqrt{r}+(m / r)^{k-1} m\right)=O\left(m^{k /(2 k-1)} n^{(2 k-2) /(2 k-1)}\right) .
\end{aligned}
$$

\section{Spanning trees with low crossing number in the plane}

In the forthcoming proof of Theorem 1.3, instead of constructing a geometric spanning tree directly, it will be more natural to construct an arcwise connected set $X$, made of segments and algebraic arcs, that has a low crossing number and contains the given point set $P$. Here we say that a set $X \subseteq \mathbb{R}^{d}$ has crossing number at most $k$ if each line, possibly with finitely many exceptions, intersects $X$ in at most $k$ points. (It is easy to check that for a geometric spanning tree, this new definition is equivalent to the earlier one.)

The following lemma allows us to convert such an $X$ into a geometric spanning tree. Although we are not aware of an explicit reference for the statement we need, most of the ideas of the proof appear in the literature in some form.

Lemma 5.1 Let $P$ be a set of $n$ points in the plane, and let $X$ be an arcwise connected set containing $P$, with crossing number at most $k$. Then there exists a (geometric) spanning tree of $P$ whose edges are straight segments and whose crossing number is at most $2 k$.

Proof. In the first stage of the proof we build a Steiner tree $S$ for $P$, whose edges are arcs contained in $X$. We order the points of $P$ arbitrarily, into a sequence $p_{1}, p_{2}, \ldots, p_{n}$. We set $S_{1}=\left\{p_{1}\right\}$, and, having built a Steiner tree $S_{i} \subseteq X$ for $\left\{p_{1}, \ldots, p_{i}\right\}$, we choose an arc $\alpha_{i}$ connecting $p_{i+1}$ to some point $q_{i}$ of $S_{i}$, in such a way that $\alpha_{i} \cap S_{i}=\left\{q_{i}\right\}$. Then we set $S_{i+1}:=S_{i} \cup \alpha_{i}$. Having reached $i=n$, we set $S:=S_{n}$; see Fig. 11. The crossing number of $S$ is at most $k$ since $S \subseteq X$.

In the second stage, we replace arcs by straight segments. Namely, the points $q_{j}$ divide $S$ into finitely many subarcs, and we replace each of them by a straight segment connecting its endpoints. It is easily seen (and standard) that the crossing number does not increase. This yields a Steiner tree for $P$ whose edges are straight segments.

In the third and last stage, we eliminate the Steiner points and obtain a spanning tree, at the price of at most doubling the crossing number. This is done by performing an inorder traversal of the tree, starting from some arbitrary root vertex, tracing each edge in both directions, skipping over the Steiner points, connecting each pair of consecutively visited points of $P$ by a straight segment, and finally eliminating cycles in the resulting tour.

The main step in the proof of Theorem 1.3 is the following lemma. 

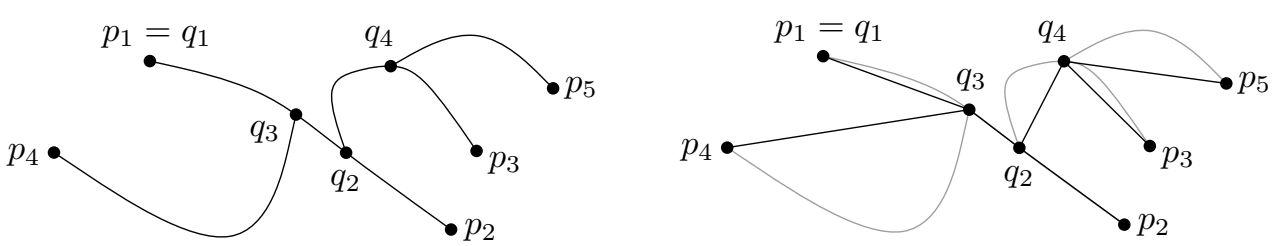

Figure 1: Illustrating the proof of Lemma 5.1] Left: Building a Steiner tree from arcs. Right: Shortcutting the arcs into segments.

Lemma 5.2 Let $P$ be a set of $n$ points in the plane. Then there exists a set $X \subseteq \mathbb{R}^{2}$ that contains $P$, has at most $n / 2$ arcwise connected components, and with crossing number $O(\sqrt{n})$.

Proof. If $n$ is below a suitable constant, we can interconnect the points of $P$ by an arbitrary geometric spanning tree, and so we may assume that $n$ is large.

We apply the polynomial partitioning theorem (Theorem 2.6), to obtain an $r$-partitioning polynomial $f$ for $P$, with $r$ as large as possible but so that $Z:=Z(f)$ is guaranteed to have at most $n / 2$ connected components. By Theorem 2.6. we have $\operatorname{deg}(f)=O(\sqrt{r})$, and so, by Harnack's theorem (Theorem 2.3), we can afford to take $r=n / c$ for a suitable constant $c$.

Then, for every $p \in P$ not lying in $Z$, we pick a straight segment $\sigma_{p}$ connecting $p$ to a point of $Z$ (and otherwise avoiding $Z$ ). We let $X:=Z \cup \bigcup_{p \in P \backslash Z} \sigma_{p}$. Clearly, $X$ has at most $n / 2$ components, and it remains to bound its crossing number.

Let $\ell$ be a line that is not contained in $Z$ and that does not contain any of the segments $\sigma_{p}$ (these conditions exclude only finitely many lines). It intersects $Z$ in at $\operatorname{most} \operatorname{deg}(f)=O(\sqrt{n})$ points, and so it remains to bound the number of the segments $\sigma_{p}$ intersected by $\ell$.

Since $f$ is an $r$-partitioning polynomial for $P$, no component of $\mathbb{R}^{2} \backslash Z$ contains more than $c$ points of $P$. The line $\ell$ meets at most $1+\operatorname{deg}(f)$ components, and so it intersects at most $c(1+\operatorname{deg}(f))=O(\sqrt{n})$ of the segments $\sigma_{p}$. The lemma is proved.

Proof of Theorem 1.3. In view of Lemma 5.1, it suffices to construct an arcwise connected set $X$ containing $P$, with crossing number $O(\sqrt{n})$. To this end, we apply Lemma 5.2 recursively.

We construct a sequence $B_{0}, B_{1}, B_{2}, \ldots$ of sets, such that each $B_{i}$ contains $P$ and has at most $n / 2^{i}$ arcwise connected components. We begin with $B_{0}:=P$, and, having constructed $B_{i}$, we choose a point in each of its components, which yields a set $R_{i}$ of at most $n / 2^{i}$ points. Lemma 5.2 then provides us with a set $X_{i} \supseteq R_{i}$ with at most $n / 2^{i+1}$ components and crossing number $O\left(\sqrt{n / 2^{i}}\right)$. We set $B_{i+1}:=B_{i} \cup X_{i}$ and continue with the next iteration, until for some $i_{0}$ we reach an arcwise connected $B_{i_{0}}$, which we use as $X$. The crossing numbers of the $X_{i}$ are bounded by a geometrically decreasing sequence, and so $X$ has crossing number bounded by its sum, which is $O(\sqrt{n})$, as required.

\section{Spanning trees in higher dimensions}

Here we prove the higher-dimensional generalization of Theorem 1.3 mentioned in the introduction.

Theorem 6.1 Every set $P$ of $n$ points in $\mathbb{R}^{d}$ admits a geometric spanning tree with crossing number (w.r.t. hyperplanes) at most $C_{d} n^{1-1 / d}$, with $C_{d}$ a sufficiently large constant depending on $d$. 
When one tries to extend the planar proof from Section 5, with the appropriate higher-dimensional analogs of the polynomial partition lemma and Harnack's theorem (discussed below), a problem arises when almost all the points of $P$ happen to lie on the zero set $Z(f)$ of the partitioning polynomial. (This situation seems hard to avoid - for example, $P$ may lie on a low-degree algebraic variety, in which case the zero set of each of the bisecting polynomials would simply coincide with this variety.)

In the planar case this did not matter, since we could use $Z(f)$ itself as a part of the connecting set $X$. However, in higher dimension, we cannot take all of $Z(f)$ (which is typically a $(d-1)$ dimensional object), so we would still need to construct a suitable connecting set with low crossing number within $Z(f)$.

Fortunately, the spanning tree problem behaves well with respect to small perturbations. Namely, it is easy to see (and well known) that the crossing number of a geometric spanning tree cannot change by a (sufficiently small) perturbation of its vertex set, and this will allow us to avoid the situation with too many points on $Z(f)$. Before carrying out this plan, we first summarize and review the additional tools we need, beyond those already covered.

\subsection{Additional tools}

The polynomial ham sandwich theorem (Theorem 2.5) and the polynomial partitioning theorem (Theorem 2.6) immediately generalize to $\mathbb{R}^{d}$, with the $d$-variate $r$-partitioning polynomial $f$ having degree $O\left(r^{1 / d}\right)$ (this relies on the fact that the number of monomials of degree $D$ in $d$ variables is $\left(\begin{array}{c}D+d \\ d\end{array}\right)$, so the degree will be the smallest integer satisfying $\left.\left(\begin{array}{c}D+d \\ d\end{array}\right)-1 \geq r\right)$.

We will also need a kind of generalization of Harnack's theorem, dealing with components of the complement of $Z(f)$, rather than with the components of $Z(f)$ :

Lemma 6.2 Let $f$ be a real polynomial of degree $D$ in $d$ variables. Then the number of connected components of $\mathbb{R}^{d} \backslash Z(f)$ is at most $6(2 D)^{d}$.

This follows, for example, from Warren [37, Theorem 2] (also see [4] for an exposition, and [2] for a neatly simplified proof).

We also note that if $f$ is as in Lemma 6.2 and $h$ is a hyperplane in $\mathbb{R}^{d}$, then $h \backslash Z(f)$ has at most $6(2 D)^{d-1}$ connected components, and consequently, $h$ intersects at most that many components of $\mathbb{R}^{d} \backslash Z(f)$. Indeed, this is clear from Lemma 6.2 if $h$ is the coordinate hyperplane $x_{d}=0$, and the general case follows by a linear transformation of coordinates.

\subsection{A general position lemma}

We need the following lemma, which is probably known, but unfortunately we do not have a reference at the moment.

Lemma 6.3 Let $d, D$ be given integers, and let $k:=\left(\begin{array}{c}D+d \\ d\end{array}\right)-1$. Let $P=\left(p_{1}, \ldots, p_{k+1}\right)$ be an ordered $(k+1)$-tuple of points in $\mathbb{R}^{d}$. Let us call $P$ exceptional if it is contained in the zero set of a nonzero $d$-variate polynomial of degree at most $D$. Then there is a nonzero polynomial $\psi=\psi_{d, D}$ with integer coefficients in the variables $z_{i j}, 1 \leq i \leq k+1,1 \leq j \leq d$, such that all exceptional $(k+1)$-tuples $\left(p_{1}, \ldots, p_{k+1}\right)$ belong to the zero set of $\psi$ (that is, if we set $z_{i j}$ to the $j$ th coordinate of $p_{i}$, for all $i, j$, then $\psi$ evaluates to 0$)$. 
Proof. The value of $k$ in the lemma is the number of nonconstant monomials of degree at most $D$ in the $d$ variables $x_{1}, \ldots, x_{d}$. Let $\mu_{1}, \mu_{2}, \ldots, \mu_{k}$ be an enumeration of these monomials in some fixed order.

It is convenient to phrase the argument using the Veronese map $\Phi: \mathbb{R}^{d} \rightarrow \mathbb{R}^{k}$, which we encountered in Section 2.2 for the special case $d=2$. For $x=\left(x_{1}, \ldots, x_{d}\right) \in \mathbb{R}^{d}$, we can write $\Phi(x)=\left(\mu_{i}(x) \mid i=1,2, \ldots, k\right) \in \mathbb{R}^{k}$.

As in the proof of Theorem 2.5, the zero set $Z(f)$ of a polynomial $f$ of degree at most $D$ can be written as $\Phi^{-1}(h)$, where $h$ is a suitable hyperplane in $\mathbb{R}^{k}$. Thus, the condition for a sequence $P=\left(p_{1}, \ldots, p_{k+1}\right)$ of points in $\mathbb{R}^{d}$ to be exceptional is equivalent to the $k+1$ points $\Phi\left(p_{1}\right), \ldots, \Phi\left(p_{k+1}\right)$ lying on a common hyperplane in $\mathbb{R}^{k}$.

The condition that $k+1$ points $q_{1}, \ldots, q_{k+1}$ in $\mathbb{R}^{k}$ lie on a common hyperplane can be expressed by the vanishing of a suitable determinant in the coordinates of $q_{1}, \ldots, q_{k+1}$. Namely, it is equivalent to $\operatorname{det}(A)=0$, where

$$
A=A\left(q_{1}, \ldots, q_{k+1}\right)=\left(\begin{array}{ccccc}
1 & q_{11} & q_{12} & \cdots & q_{1 k} \\
1 & q_{21} & q_{22} & \cdots & q_{2 k} \\
& \vdots & & \vdots & \\
1 & q_{(k+1) 1} & q_{(k+1) 2} & \cdots & q_{(k+1) k}
\end{array}\right) .
$$

We define the desired polynomial $\psi$ by

$$
\psi=\psi\left(z_{11}, \ldots, z_{(k+1) d}\right):=\operatorname{det}\left(A\left(\Phi\left(z_{1}\right), \Phi\left(z_{2}\right), \ldots, \Phi\left(z_{k+1}\right)\right)\right),
$$

where $z_{i}=\left(z_{i 1}, \ldots, z_{i d}\right)$. Clearly, $\psi$ has integer coefficients, and by the above, it vanishes on all exceptional sequences; it remains to verify that it is not identically 0 .

Assuming the contrary, it means that the images of any $k+1$ points under the Veronese map lie on a common hyperplane in $\mathbb{R}^{d}$. This in turn implies that all of $\Phi\left(\mathbb{R}^{d}\right)$ is contained in a hyperplane. But the $\Phi$-preimage of every hyperplane is the zero set of some nonzero polynomial, and thus it cannot be all of $\mathbb{R}^{d}$ (extending the observation in the proof of Lemma 2.2 to higher dimensions) 7 The resulting contradiction proves the lemma.

\subsection{Proof of Theorem 6.1}

Given a finite point set $P \subset \mathbb{R}^{d}$, we first perturb each point slightly, obtaining a new set $P^{\prime}$, for which we may assume that the coordinates of its points are algebraically independent (i.e., they do not satisfy any nontrivial polynomial equation with integer coefficients) 8

In particular, for every $D=1,2, \ldots$, if we set $k:=\left(\begin{array}{c}D+d \\ d\end{array}\right)-1$ as in Lemma 6.3, then no $(k+1)$ tuple of points of $P^{\prime}$ is contained in $Z(f)$, for any nonzero polynomial $f$ of degree at most $D$.

By the observation mentioned at the beginning of Section 6, it suffices to exhibit a geometric spanning tree with crossing number $O\left(n^{1-1 / d}\right)$ for $P^{\prime}$.

\footnotetext{
${ }^{7}$ Another way to see that $\psi$ is not identically zero is to consider two terms $t_{1}$ and $t_{2}$ in the expansion of $\operatorname{det}\left(A\left(\Phi\left(z_{1}\right), \Phi\left(z_{2}\right), \ldots, \Phi\left(z_{k+1}\right)\right)\right)$. There is a row $i$ of $A\left(\Phi\left(z_{1}\right), \Phi\left(z_{2}\right), \ldots, \Phi\left(z_{k+1}\right)\right)$ from which $t_{1}$ and $t_{2}$ contain different elements. It follows that $t_{1}$ and $t_{2}$ must contain a different power of one of the variables $z_{i 1}, \ldots, z_{i d}$. We get that all terms in the expansion of $\operatorname{det}\left(A\left(\Phi\left(z_{1}\right), \Phi\left(z_{2}\right), \ldots, \Phi\left(z_{k+1}\right)\right)\right)$ are are different monomials, so $\psi$ cannot be identically zero.

${ }^{8}$ The existence of such $P^{\prime}$ is well known and follows, e.g., by a standard measure argument via Sard's theorem (which guarantees that the zero set of every nonzero multivariate polynomial has zero measure; see, e.g., [31, 32]).
} 
Moreover, it suffices to show that there exists a geometric graph $G$ on the vertex set $P^{\prime}$ with at most $n / 2$ components and with crossing number $O\left(n^{1-1 / d}\right)$; the existence of the desired spanning tree then follows by recursion on the size of $P^{\prime}$ (as in the proof of the planar case).

So we set $r:=n / c$ for a sufficiently large constant $c>0$, and construct an $r$-partitioning polynomial $f$ for $P^{\prime}$, of degree $D=O\left(r^{1 / d}\right)$; thus, no component of $\mathbb{R}^{d} \backslash Z$ contains more than $c$ points of $P^{\prime}$, where $Z=Z(f)$.

By the algebraic independence of $P^{\prime}$, and by Lemma 6.3, $Z$ contains fewer than $\left(\begin{array}{c}D+d \\ d\end{array}\right)=O(r)$ points of $P^{\prime}$, with a constant of proportionality depending only on $D$. For $c$ sufficiently large, we thus have $\left|P_{0}^{\prime}\right| \leq \frac{n}{4}$, where $P_{0}^{\prime}:=P^{\prime} \cap Z$. By Lemma 6.2, we may also assume that for $c$ sufficiently large, $\mathbb{R}^{d} \backslash Z$ has at most $\frac{n}{4}$ components.

For each component $U$ of $\mathbb{R}^{d} \backslash Z$, we now interconnect the points of $U \cap P^{\prime}$ by an arbitrary geometric spanning tree $T_{U}$. The geometric graph $G$ is the union of all the trees $T_{U}$ and the points of $P_{0}^{\prime}$ (which appear as isolated vertices in $G$ ). The number of connected components of $G$ is at most $\frac{n}{2}$ (one for each $T_{U}$ and one for each point of $P_{0}^{\prime}$ ). It remains to bound its crossing number.

To this end, let $h$ be a hyperplane avoiding $P^{\prime}$, and let us consider an edge $\{p, q\}$ of $G$ crossed by $h$. This edge belongs to some $T_{U}$, and so the points $p$ and $q$ lie in the same component $U$ of $\mathbb{R}^{d} \backslash Z$. Considering an arc $\alpha \subset U$ connecting $p$ to $q$, we see that $h$ has to intersect $\alpha$ and thus $U$ too. By the remark following Lemma 6.2, $h$ intersects at most $O\left(D^{d-1}\right)=O\left(n^{1-1 / d}\right)$ components of $\mathbb{R}^{d} \backslash Z$, and within each such component $U$ it meets at most $c$ edges of $G$ (that is, of $T_{U}$ ). Hence the crossing number of $G$ is $O\left(n^{1-1 / d}\right)$, as claimed.

\section{Conclusion}

We regard this paper as an initial stepping stone in the development of applications of the new algebraic machinery of Guth and Katz. It is encouraging that this technique can replace more traditional approaches and yield simpler proofs of central theorems in combinatorial geometry.

Of course the real challenge is to use the techniques to obtain improved solutions to other "hard Erdős problems in discrete geometry" (borrowing from the title of [34]), as Guth and Katz themselves did, first for the joints problem in [15] and then for the harder distinct distances problem in [16]. There is a long list of candidate problems, of varying degree of difficulty. Perhaps the hardest in the list is the planar unit distances problem of Erdős: What is the maximum possible number of unit distances determined by a set of $n$ points in the plane? This problem seems to require an algebraic approach, mainly because the best known upper bound, $O\left(n^{4 / 3}\right)$, is known to be tight if the norm is not Euclidean, as shown by Valtr [36].

In closing, one should note that the algebraic approach used in this paper has also some disadvantages. For one, it seems to require the objects to be algebraic or semialgebraic. For example, the Szemerédi-Trotter theorem can easily be extended to yield the same bound on the number of incidences of points and pseudolines, using, e.g., the combinatorial proof technique of Székely [34], but such an extension does not seem to follow from the polynomial partitioning technique. The same situation occurs in the setup of Theorem 1.2, where the general situation considered there can be handled by traditional combinatorial tools, but not by the algebraic machinery, which can only establish weaker variants, like the one in Theorem 4.1. Perhaps some abstract version of polynomial partitions, yet to be discovered, might combine the advantages of both approaches. 


\section{Acknowledgements.}

The authors wish to thank Roel Apfelbaum and Sariel Har-Peled for useful exchanges of ideas that were helpful in the preparation of this paper.

\section{References}

[1] P. K. Agarwal and J. Matoušek, On range searching with semialgebraic sets, Discrete Comput. Geom. 11 (1994), 393-418.

[2] Y. Akama, K. Irie, A. Kawamura and Y. Uwano, VC dimensions of principal component analysis, Discrete Comput. Geom. 44 (2010), 589-598.

[3] T. Asano, M. de Berg, O. Cheong, L. J. Guibas, J. Snoeyink and H. Tamaki, Spanning trees crossing few barriers, Discrete Comput. Geom. 30 (2003), 591-606.

[4] S. Basu, R. Pollack, and M.-F. Roy, Algorithms in Real Algebraic Geometry, Algorithms and Computation in Mathematics 10, Springer-Verlag, Berlin, 2003.

[5] T. M. Chan, Optimal partition trees, In Proc. 26th Ann. ACM Sympos. Comput. Geom., pages $1-10,2010$.

[6] B. Chazelle, Cuttings, in Handbook of Data Structures and Applications (D. Mehta and S. Sahni, eds.), chap. 25, Chapman and Hall/CRC Press, Boca Raton, FL, 2005.

[7] B. Chazelle and E. Welzl, Quasi-optimal range searching in spaces of finite VC-dimension, Discrete Comput. Geom. 4 (1989), 467-489.

[8] K. Clarkson, H. Edelsbrunner, L. Guibas, M. Sharir and E. Welzl, Combinatorial complexity bounds for arrangements of curves and spheres, Discrete Comput. Geom. 5 (1990), 99-160.

[9] D. Cox, J. Little and D. O'Shea, Using Algebraic Geometry, 2nd edition, Springer-Verlag, Heidelberg, 2004.

[10] D. Cox, J. Little and D. O'Shea, Ideals, Varieties, and Algorithms: An Introduction to Computational Algebraic Geometry and Commutative Algebra (3rd edition), Springer-Verlag, Heidelberg, 2007.

[11] Gy. Elekes, Sums versus products in number theory, algebra and Erdős geometry, in G. Halász et al. editors, Paul Erdős and His Mathematics, J. Bolyai Math. Soc., Budapest, 2001.

[12] Gy. Elekes, H. Kaplan and M. Sharir, On lines, joints, and incidences in three dimensions, $J$. Combinat. Theory, Ser. A 118 (2011), 962-977. Also in arXiv:0905.1583.

[13] Gy. Elekes and M. Sharir, Incidences in three dimensions and distinct distances in the plane, in Proc. 26th Annu. ACM Sympos. Comput. Geom., pages 413-422, 2010.

[14] P. Erdős, On a set of distances of $n$ points, Amer. Math. Monthly 53 (1946), 248-250.

[15] L. Guth and N. H. Katz, Algebraic methods in discrete analogs of the Kakeya problem, Advances Math. 225 (2010), 2828-2839. Also in arXiv:0812.1043v1. 
[16] L. Guth and N. H. Katz, On the Erdős distinct distances problem in the plane, arXiv:1011.4105.

[17] C. G. A. Harnack, Über Vieltheiligkeit der ebenen algebraischen Curven, Math. Ann. 10 (1876), 189-199.

[18] S. Har-Peled, Approximating spanning trees with low crossing numbers, preprint, arXiv:0907.1131.

[19] N. H. Katz and G. Tardos, A new entropy inequality for the Erdös distance problem, in Towards a Theory of Geometric Graphs (J. Pach, ed.), Contemporary Mathematics 342, AMS, Providence, RI, 2004, 119-126.

[20] C. Knauer, H.R. Tiwary, D. Werner, On the computational complexity of Ham-Sandwich cuts, Helly sets, and related problems, In Proc. 28th Int. Sympos. on Theoretical Aspects of Computer Science (STACS), 2011, to appear.

[21] T. Kővári, V. Sós, and P. Turán, On a problem of K. Zarankiewicz. Coll. Math. 3 (1954), $50-57$.

[22] J. Matoušek, Efficient partition trees, Discrete Comput. Geom. 8 (1992), 315-334.

[23] J. Matoušek, Improved upper bounds for approximation by zonotopes, Acta Math. 177 (1996), $55-73$.

[24] J. Matoušek, Lectures on Discrete Geometry, Springer Verlag, Heidelberg, 2002.

[25] J. Matoušek, Using the Borsuk-Ulam Theorem, Lectures on Topological Methods in Combinatorics and Geometry Series, Springer Verlag, Heidelberg, 2003.

[26] J. Matoušek, E. Welzl, and L. Wernisch, Discrepancy and $\varepsilon$-approximations for bounded VCdimension, Combinatorica 13 (1993), 455-466.

[27] J. Pach and P. K. Agarwal, Combinatorial Geometry, Wiley-Interscience, New York, 1995.

[28] J. Pach and M. Sharir, Repeated angles in the plane and related problems, J. Combinat. Theory Ser. A 59 (1992), 12-22.

[29] J. Pach and M. Sharir, On the number of incidences between points and curves, Combinat. Probab. Comput. 7 (1998), 121-127.

[30] J. Pach and M. Sharir, Geometric incidences, in Towards a Theory of Geometric Graphs (J. Pach, ed.), Contemporary Mathematics, Vol. 342, Amer. Math. Soc., Providence, RI, 2004, pp. 185-223.

[31] A. Sard, The measure of the critical values of differentiable maps, Bull. Amer. Math. Soc. 48 (1942), 883-890.

[32] S. Sternberg, Lectures on Differential Geometry, Prentice Hall, Englewood Cliffs, NJ, 1964.

[33] A. H. Stone and J. W. Tukey, Generalized sandwich theorems, Duke Math. J. 9 (1942), 356359. 
[34] L. Székely, Crossing numbers and hard Erdős problems in discrete geometry, Combinat. Probab. Comput. 6 (1997), 353-358.

[35] E. Szemerédi and W. T. Trotter, Extremal problems in discrete geometry, Combinatorica 3 (1983), 381-392.

[36] P. Valtr, Strictly convex norms allowing many unit distances and related touching questions, manuscript, Charles University, Prague 2005.

[37] H. E. Warren, Lower bound for approximation by nonlinear manifolds, Trans. Amer. Math. Soc. 133 (1968), 167-178.

[38] E. Welzl, Partition trees for triangle counting and other range searching problems, in Proc. 4th Annu. ACM Sympos. Comput. Geom., pages 23-33, 1988.

[39] E. Welzl, On spanning trees with low crossing numbers, in Data Structures and Efficient Algorithms, Lecture Notes in Computer Science, vol. 594, Springer Verlag, 1992, pp. 233-249. 